\title{
Equipment
}

\section{Lightwand intubation: I - A new lightwand device}

Orlando Ricardo Hung MD FRCPC, Ronald D. Stewart MD FRCPC*
Tracheal intubation under direct vision using a laryngoscope can be challenging and difficult even in experienced hands. Transillumination of the sofi tissues of the neck using a lightedstylet (ightwand) is one of many effective altermative intubating techniques developed during the past several decades. While many versions of lightwand have been available, each has its limitations. A newly developed lightwand (Trachlight(i)) has incorporated many design modifications. It has a brighter bulb, permitting intubation under ambient light in most cases. $A$ retractable stiff wire within the wand adds flexibility to facilitate both oral and nasal intubation. The flexible wand also allows visual (transillumination) guide to proper placement of the tip of the tracheal tube in the trachea. The Trachlight (if) does have some limitations. It is a light-guided technique in which there is no direct visualization of the upper airway structures. It should be avoided in patients with known anatomical abnormalities of the upper airway and used with caution in patients in whom transillumination of the anterior neck may not be achieved adequately. As with any intubating technique, successful intubation using the Trachlight (1) relies on the preparation of the patient and the operator's skill and experience.

Même entreprise par des personnes expérimentées, lintubation sous vision directe avec un laryngoscope peut être laborieuse. La transillumination des tissus du cou avec un mandrin lumineux constitue une des techniques de rechange développées

\section{Key words}

ANAESTHETIC EQUIPMENT: Lightwand, lighted-stylet; INTUBATION, TRACHEAL: technique, Lightwand.

From the Departments of Anaesthesia and Emergency Medicine*, Dalhousie University, Halifax, Nova Scotia, Canada.

Address correspondence to: Dr. Orlando R. Hung,

Department of Anaesthesia, Victoria General Hospital,

Halifax, Nova Scotia, Canada, B3H 2 Y9.

Supported, in part, by a Grant from Laerdal Research

Foundation, Armonk, NY.

Accepted for publication 19th May, 1995. au cours des dernières décennies. Bien que plusieurs versions du mandrin lumineux soient disponibles, elles ont toutes leurs limites. Un nouveau mandrin lumineux (Trachlight $(0)$ ) incorpore plusieurs modifications au design original. L'éclairage est intense, ce qui permet l'intubation à la lumière ambiante dans la plupart des cas. Un stylet rétractile à lintérieur du mandrin ajoute de la flexibilité ce qui facilite lintubation orale et nasale. Le mandrin flexible permet aussi de guider visuellement (par transillumination) le bout de la canule endotrachéale dans la trachée. Le Trachlight ${ }^{(0)}$ a aussi ses limites. I s'agit diune technique d'orientation par la lumière qui ne perm et pas de visualiser directement les structures des voies aériennes supérieures. Elle devrait être évitée dans les cas d'anomalies connue de l'anatomie des voies aériennes supérieures et utilisée avec prudence lorsque la transilumination des structures antérieures du cou ne peut être réalisée correctement. Comme pour toutes les techniques dintubation, le succès dépend de la préparation du patient, et de l'expérience et de lhabileté de lintubateur.

Despite more than $80 \mathrm{yr}$ of direct-vision laryngoscopy, accurate and prompt placement of the endotracheal tube (ETT) remains a considerable challenge in some patients, even in experienced hands. This is especially true in "unprepared" patients, or patients requiring emergency tracheal intubation. It has been estimated that between 1-3\% of surgical patients have "difficult airways," making laryngoscopic intubation difficult and sometimes impossible. ${ }^{1}$ Because of these difficulties, alternative intubating techniques have been developed. Intubation using a lighted-stylet (lightwand) is one of many. effective intubating techniques. The objectives of this paper are to review the concept of transillumination, to introduce a new lightwand device (Trachlight $(\mathbb{0})$ ), and to describe the technique of intubation using this new lightwand.

Intubation using the principle of transillumination The technique of transillumination was first described by Yamamura et al. in 1959 when they reported the use of a lightwand for nasotracheal intubation. ${ }^{2}$ The light- 
wand uses the principle of transillumination of the soft tissues of the anterior neck to guide the tip of the ETT into the trachea. The lightwand is placed inside an ETT during intubation, so that the bulb of the lightwand produces an intense light at the tip of the ETT. When the tip of the ETT enters the glottic opening, a well defined circumscribed glow can readily be seen in the anterior neck slightly below the thyroid prominence. However, if the tip of the tube is placed in the oesophagus, the transmitted glow is diffuse and may not be detected easily under ambient lighting conditions. If the tip of the ETT is placed in the vallecula, the light glow is diffuse and is slightly above the thyroid prominence. Using this principle, the lightwand can be used to guide the tip of the ETT easily and safely into the trachea without the use of a laryngoscope.

During the past decade, several versions of lightwand have been introduced. Although these devices have proved to be effective and safe for tracheal intubation, ${ }^{3-6}$ they have some limitations: (1) the intensity of the light bulb illumination is insufficient to produce adequate transillumination under ambient light; (2) a clamp or connector to secure the ETT onto the lightwand is not incorporated in some of the lightwands, making it necessary to tape the ETT to the devices; (3) because of the rigidity of the wand portion of the device, the ETT is frequently dislodged from the glottic opening while withdrawing the lightwand from the ETT following intubation. In addition, following the withdrawal of the lightwand from the ETT, it is impossible to detect the location of the tip of the ETT. This may lead to inadvertent oesophageal intubation or more likely, right bronchial intubation.

\section{A new lightwand device (Trachlight(1))}

A new lightwand device (Trachlight(10) - Laerdal Medical Inc., Armonk, New York) has recently been introduced. The Trachlight@ consists of three parts: a reusable handle, a flexible wand, and a stiff retractable stylet (Figure 1). The Trachlight $(5)$ requires three triple " $A^{n}$ alkaline batteries which are encased within the handle and can be changed easily as required. A locking clamp is located on the front of the handle, which accepts and secures a standard ETT connector. The wand consists of a flexible plastic shaft with a bright light bulb affixed at the distal end. The light beam shines with a forward projection and with a wider angle from the tip of the lightwand (Figure 2). This provides better illumination of the soft tissues of the neck during intubation using the lightwand. With the improved bulb technology, the light emitted by the Trachlight $($ is is extremely bright with minimal heat production (a maximum surface temperature of approximately $60^{\circ} \mathrm{C}$ ). After $30 \mathrm{sec}$ illumination, the light bulb blinks off and on to further minimize heat pro-

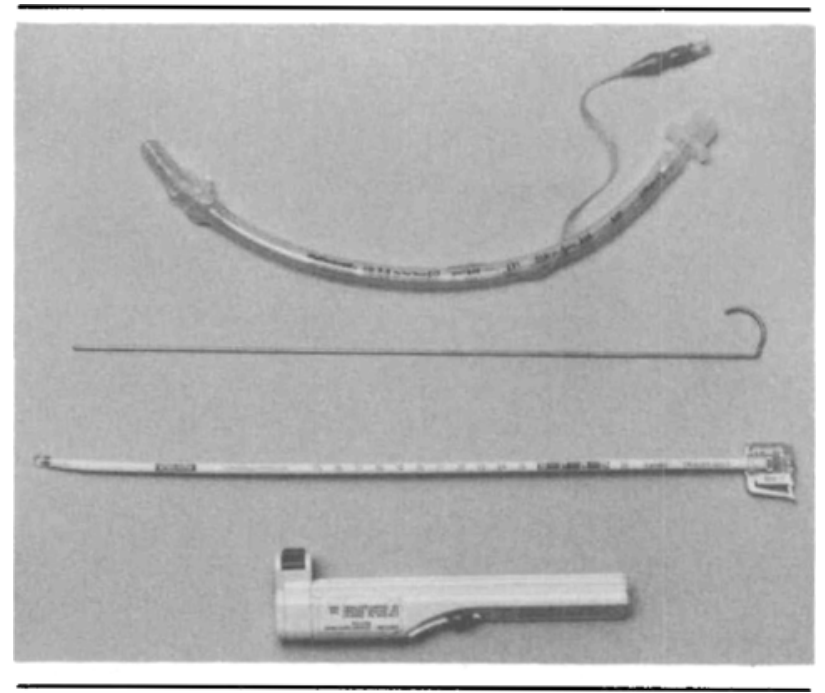

FIGURE 1 The Trachlight $(1)$ consists of three parts: a handte, a flexible wand, and a stiff internal stylet.

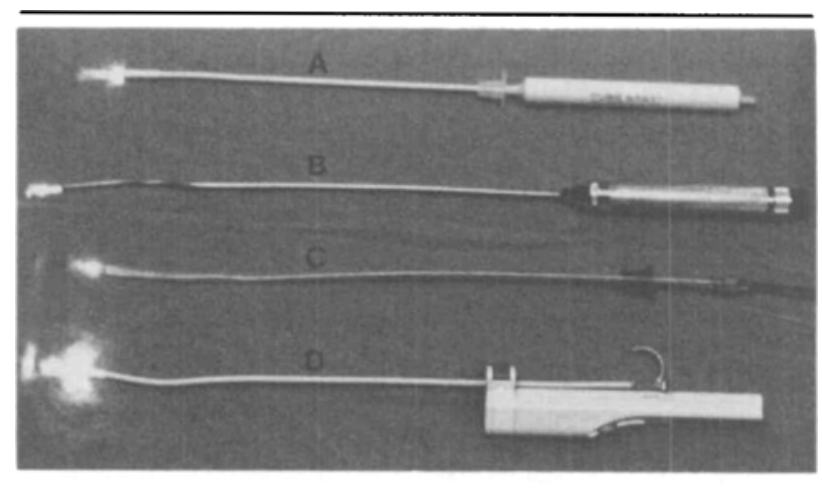

FIGURE 2 The light beam shines only forward from the tip of the three older versions of lightwands: (A) Tubestat ${ }^{(1)}$, (B) Fibre Optic Lighted-Intubation Stilette, (C) Fibreoptic Lighted Stylet. The light beam of the Trachlight $\Theta$ shines forward and laterally with a wider angle. This will provide better transillumination of the soft tissues of the neck during intubation using the Trachlight $($ ).

duction. Since the tip of the light bulb is encased within the ETT, thermal injury during intubation is unlikely. The improved light source of the Trachlight 10 permits intubation under ambient lighting conditions, usually making it unnecessary to dim the room light.

Affixed to the proximal end of the wand is a rigid plastic connector with a release arm allowing its connection to the grooves in the wand handle. This connector allows adjustment of the wand along the handle by depressing the release arm and gliding it along the handle to accommodate ETT's of varying lengths. Enclosed within the wand is a stiff, but malleable, retractable stylet. The retractable stylet gives sufficient stiffness to the device, allowing the wand to be shaped in a "field-hockey 


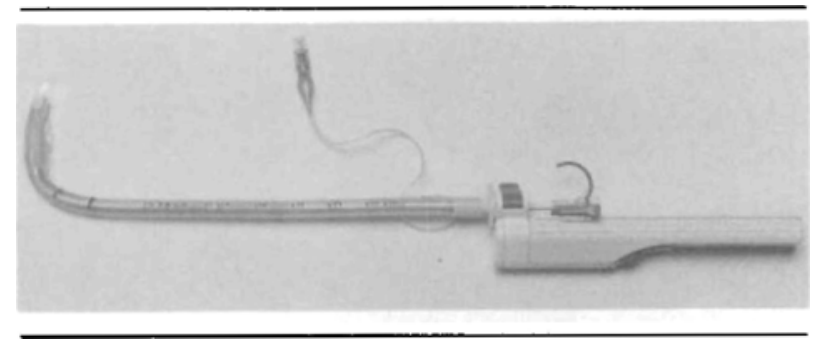

FIGURE 3 Following the insertion of the wand of the Trachlight ${ }^{\oplus}$ into the endotracheal tube, a $90^{\circ}$ bend is made at the proximal end of the tube to form the "field-hockey stick" configuration.

stick" configuration (Figure 3). This shape enhances manoeuvrability during intubation and facilitates the placement of the ETT into the glottic opening. However, once the tip of the ETT enters the glottic opening, the "fieldhockey stick" configuration impedes further advancement of the tube into the trachea. Retraction of the stiff stylet produces a pliable ETT-Trachlight(0) unit (ETT-TL), allowing advancement into the trachea. The retractable stylet gives flexibility to the lightwand, improving its effectiveness in placing the ETT in the trachea during oral and nasal intubations. The flexible wand also allows a proper placement of the tip of the ETT in the trachea by observing the well circumscribed glow of the transilluminated light at the level of the sternal notch. At the sternal notch, the tip of the endotracheal tube is about half-way between the vocal cords and the carina.?

\section{Technique of Trachlight $($ iS intubation}

It should be emphasized that the success of endotracheal intubation relies more on the intubator's experience and skill than on the intubation device itself. Regardless of the intubation tool, the success of any intubation depends on proper patient selection, meticulous preparation, good technique and regular practice.

\section{Preparation of the Trachlight $($ )}

Lubrication of the stiff internal stylet of the wand using silicone fluid ensures its easy retraction during intubation. The internal wall of the ETT should be lubricated with a water soluble lubricant to facilitate the removal of the wand following ETT placement. The length of the wand is adjusted by sliding the wand along the handle, placing the light bulb at the end of the ETT without protruding beyond its tip. A bend is placed in the ETT-TL unit to a $90^{\circ}$ angle just proximal to the cuff of the tube in the shape of a "field-hockey stick" (Figure 3). When the tip of the ETT is at the glottic opening, a $90^{\circ}$ bend allows the maximum light intensity pointing to the anterior wall of the larynx, producing a well defined circumscribed glow on the surface of the skin. However, if the Trach- light(ㅇ) is bent to a $45^{\circ}$, the maximum light intensity is more likely pointing down the trachea once the ETT enters the glottic opening, making transillumination difficult. Furthermore, the ETT is more likely to be placed in the oesophagus when the Trachlight $(0)$ is bent to $45^{\circ}$.

\section{Preparation and positioning of the patient}

The intubator usually stands (or kneels down if the patient is on the floor). It is possible to use the device from the front or side of the patient enhancing its utility in the prehospital arena. In contrast to the sniffing position (flexion of the neck on the shoulders and extension at the atlanto-occipital joint) for laryngoscopic intubation, the patient's head and neck should be in a neutral or relatively extended position. The epiglottis is almost in contact with the posterior pharyngeal wall when the head is in the sniffing position, making it difficult for the Trachlight(10) to go underneath the epiglottis. However, the epiglottis is lifted off the posterior pharyngeal wall when the head is extended. In general, it is recommended to place the patient's head in the neutral or slightly extended position in the absence of potential C-spine instability. In obese patients or patients with an extremely short neck, placing a pillow under the shoulders and neck may be useful.

\section{Light source}

In most cases, the trachea can be intubated easily under ambient lighting conditions. In thin patients the light bulb is so bright that it is possible to misinterpret an oesophageal intubation as an intratracheal placement if the room light is dimmed. Therefore, it is recommnded that all intubations using the Trachlight ${ }^{(0)}$ be carried out under ambient light. Dimming room lights or shading the anterior neck should be done only in obese patients or patients with thick necks in whom transillumination may not be optimal. It is rare that a dark environment is required using the Trachlight(i). In fact, nearly $88 \%$ of intubations using the Trachlight $(10)$ can be effectively performed under ambient light with or without shading of the neck. ${ }^{8}$

\section{Technique of intubation}

\section{ORAL INTUBATION}

With the patient lying supine, the jaw is grasped and lifted upward using the thumb and index finger of the intubator's non-dominant hand (Figures 4 and 5). This lifts the tongue and epiglottis off the posterior pharyngeal wall to make a clear passage for the ETT-TL to enter the glottic opening. The non-dominant hand must be kept close to the corner of the mouth to ensure an unob- 


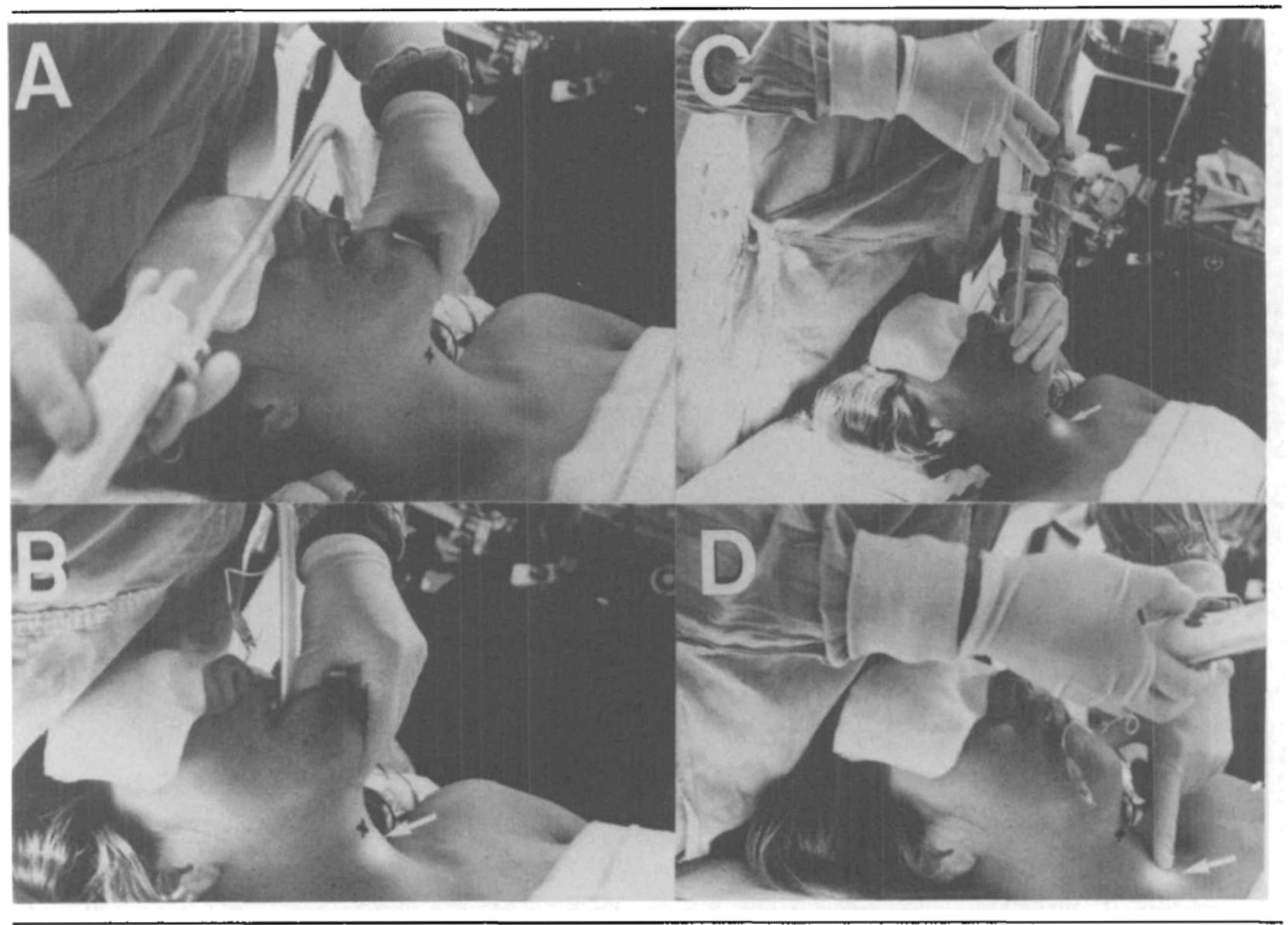

FIGURE 4 (A) The jaw is grasped by the non-dominant hand and lifted upward to elevate the tongue and epiglottis. The non-dominant hand must be kept close to the comer of the mouth to ensure an unobstructed path in the midline for the lightwand. (B) Using the dominant hand, the ETT-TL is inserted into the oropharynx. While positioned in the midline, the ETT-TL is advanced gently in a rocking motion along an imaginary arc. A bright, well defined, circumscribed glow (arrow) is seen below the thyroid prominence (marked X) when the ETT-TL enters the glottic opening. (C) The stiff internal stylet is retracted while holding the ETT close to the lips with the non-dominant hand. (D) Following retraction of the stiff internal stylet, the ETT-TL becomes pliable permitting the ETT to be advanced further into the trachea. The ETT is advanced until the glow is at the sternal notch. At the sternal notch, the tip of the ETT is about halfway between the vocal conds and the carina.

structed midline path for the ETT-TL. The ETT-TL is inserted into the midline of the oropharynx using the dominant hand. The midline position of the ETT-TL is maintained while the device is advanced gently in a rocking motion along an imaginary midline sagittal arc. When resistance is felt, the ETT-TL should be "rocked" backward and the tip redirected toward the thyroid prominence using the glow of the light as a guide. When the tip of the ETT-TL enters the glottic opening, a well defined circumscribed glow can be seen in the anterior neck slightly below the thyroid prominenoe (Figures 4 and 5). Retracting the stiff stylet approximately $10 \mathrm{~cm}$ makes the ETT-TL more pliable, allowing advancement into the trachea (Figures 4). The ETT-TL is then advanced until the glow begins to disappear at the sternal notch (Figures 4 and 5). Following release of the locking clamp, the Trachlight(응 can be removed from the ETT.
Occasionally, the circumscribed glow cannot be seen readily in the anterior neck due to anatomic features such as morbid obesity or those with short necks. Neck extension as described above may be helpful. Retraction of the breast or chest wall tissues together with indentation of the tissues around the trachea by an assistant enhances transillumination in the anterior neck. Dimming the lights is required on rare occasions.

\section{NASAL INTUBATION}

Following removal of the stiff internal stylet, the wand becomes flexible, and permits nasotracheal intubation. The ETT-TL is inserted through the nostril. With the jaw lifted upward by the non-dominant hand, the ETT$\mathrm{TL}$ is positioned in the midline and advanced gently using the light glow as a guide. When resistance is felt, the ETT-TL should be withdrawn slightly and the tip re- 


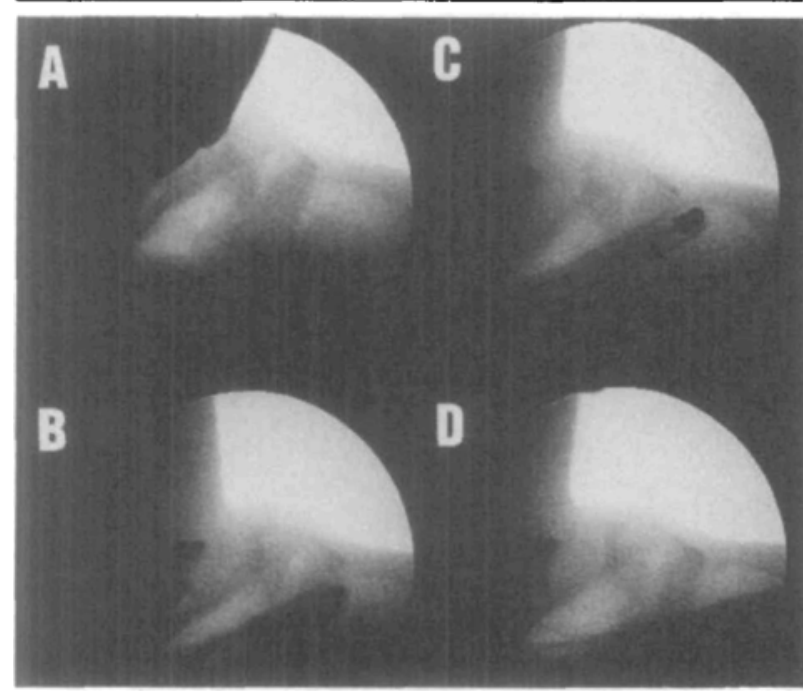

FIGURE 5 (A) Under fluoroscopy, the epiglottis (arrow) can be seen lifted off the posterior wall of the pharynx with a jaw lift. (B) When the tip enters the glottic opening, the distance between the tip of the lightwand and the surface of the skin is short. Hence, a bright circumscribed glow from the anterior neck can be readily seen, (C) When the internal stiff stylet is retracted, the ETT-TL unit becomes pliable, allowing the ETT to enter the trachea easily. (D) With the internal stylet retracted, the ETT can be advanced slowly into the trachea. Using the light glow as a guide, the tip of the ETT can readily be placed at the sternal notch.

directed toward the thyroid prominence. A faint glow seen above the thyroid prominence indicates that the tip of the ETT-TL is located in the vallecula. The jaw lift helps to elevate the epiglottis and enhances the passage of the ETT-TL under it. Alternatively, the neck of the patient can be flexed while advancing the tip of ETT-TL slowly in the midline. When the ETT-TL enters the glottic opening, a well defined, circumscribed glow is seen in the anterior neck just below the thyroid prominence (Figure 6). To ensure that the tip of the ETT-TL is located at the optimal position within the trachea, the tip of the ETT is advanced until the glow begins to disappear at the sternal notch.

\section{Advantages}

Intubation using a lightwand is a simple and easy-tolearn technique. It involves a tactile sensation of a "loss of resistance" as the tip of the ETT-TL enters the glottic opening together with visual guidance (a bright circumscribed glow below the thyroid prominence) from the soft tissues of the anterior neck. Intubation using the Trachlight $(10$ is a gentle technique and the device should not be advanced against resistance. The improved light source permits intubation under ambient light, making it possible for paramedies and other medical personnel to use the device in the field. Retraction of the internal stylet

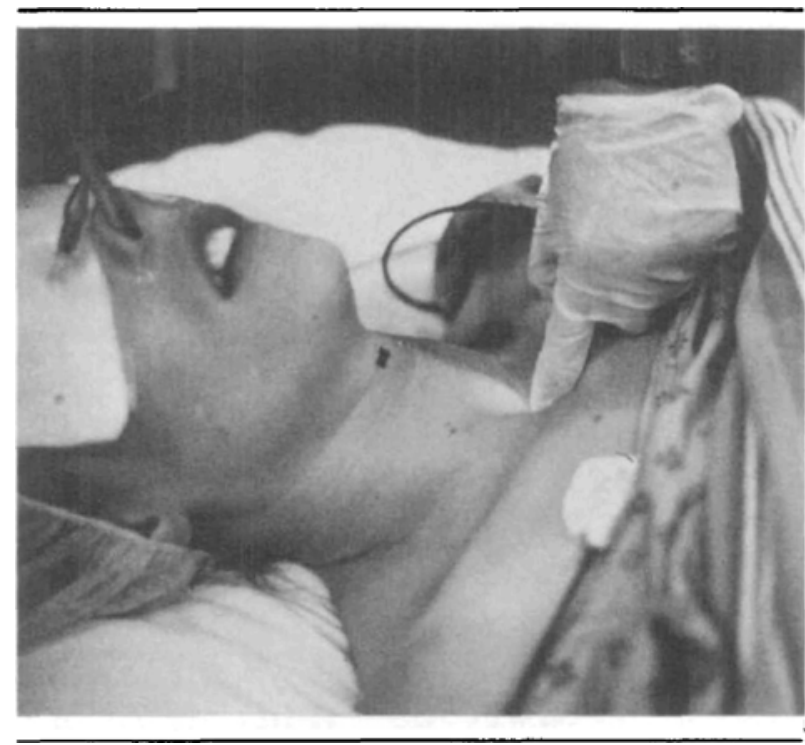

FIGURE 6 When the ETT-TL enters the glottic opening, a well defined circurnscribed glow is seen in the anterior neck just below the thyroid prominence (marked X). To ensure that the tip of the ETT-TL is located at the optimal position within the trachea, the tip of the ETT is advanced until the glow begins to disappear at the sternal notch.

increases flexibility to allow easy advancement into the glottis and trachea and to minimize laryngeal tissue injury. Following the placement of the ETT in the trachea, the pliable wand (with the stylet retracted) also allows optimal placement of the tip of the ETT within the trachea.

\section{Limitations}

While the Trachlight $(-)$ has been demonstrated to be an effective and safe device for intubation, ${ }^{8}$ it remains a lightguided technique in which there is no direct visualization of the laryngeal structures. Trachlight (iO) should be avoided in patients with known anatomical abnormalities of the upper airway, such as tumours, polyps, infection (e.g., epiglottis), trauma of the upper airway or if a foreign body is suspected in the upper airway. Trachlighte should also be used with caution in patients in whom transillumination of the anterior neck may not be adequate, such as those who are grossly obese or patients with limited neck extension. Intuitively, this light-guided technique should not be attempted with an uncooperative awake patient unless a bite block is used to prevent damage to the device or injury to the intubator.

Intubation using the Trachlight $(\oplus)$ has some risks. Stone and other investigators reported disconnection of the light-bulb from a lightwand requiring retrieval from a major bronchus. ${ }^{9}$ However, this lightwand device (Flexilum $(0)$ ) was not designed nor recommended for tracheal intubation. It is extremely unlikely that the light bulb will be detached from the Trachlight $(0$, since the light 
bulb is firmly attached to the durable plastic sheath of Trachlight (ii) in contrast to the older devices. Although rare, subluxation of the cricoarytenoid cartilage has also been reported by a study using an older version of a lightwand (Tubestat $(\mathbb{0}) .{ }^{10}$ However, with the retractable stylet, the risk of damaging the arytenoid cartilage during Trachlight (if) intubation is low. In testing the device, we have intubated the tracheas of more than six thousand patients in a four-year period, and we have not encountered any patient with symptoms and signs suggestive of cricoarytenoid subluxation.

\section{Conclusion}

The placement of an endotracheal tube in the trachea using a laryngoscope can be challenging. Transillumination of the soft tissues of the neck using a lightwand has been shown to be one of many effective alternate intubating techniques. Trachlight(1) is a new lightwand with several modifications to make it a flexible and versatile device for both nasal and oral intubations.

\section{Acknowledgement}

The authors gratefully acknowledge the technical assistance of Mr. Joseph Leary from the Audio-Visual Department of the Victoria General Hospital.

\section{References}

1 Latto IP. Management of difficult intubation. In: Latto IP, Rosen M (Eds.). Difficulties in Tracheal Intubation. London: Baillière Tindall, 1987: 99-141.

2 Yamamura $H$, Yamamoto $T$, Kamiyama $M$. Device for blind nasal intubation. Anesthesiology 1959; 20: 221.

3 Vollmer TP, Stewart RD, Paris PM, Ellis D, Berkebile PE. Use of a lighted stylet for guided orotracheal intubation in the prehospital setting. Ann Emerg Med 1985; 14: 324-8.

4 Ellis GE, Jakymec A, Kaplan RM, et al. Guided orotracheal intubation in the operating room using a lighted stylet: a comparison with direct laryngoscopic technique. Anesthesiology 1986; 64: 823-6.

5 Ainsworth $Q P$, Howells $T H$. Transilluminated tracheal intubation. Br J Anaesth 1989; 62: 494-7.

6 Weis $F R$, Hatton $M N$. Intubation by use of the light wand: experience in 253 patients. J Oral Maxillofac Surg 1989; 47: 560-77.

7 Stewart RD, LaRosee A, Kaplan RM, Ikhanipour K. Correct positioning of an endotracheal tube using a flexible lighted stylet. Crit Care Med 1990; 18: 97-9.

8 Hung OR, Pytka S, Morris IR, et al. Clinical trial of a new lightwand device (Trachlight $(1)$ ) to intubate the trachea. Anesthesiology. (in press)

9 Stone DJ, Stirt JA, Kaplan MJ, McLean WC. A complication of lightwand-guided nasotracheal intubation. Anesthesiology 1984; 61: 780-1.
10 Debo RF, Colonna D, Dewerd G, Gonzalez C. Cricoarytenoid subluxation: complication of blind intubation with a lighted stylet. Ear Nose Throat J 1989; 68: 517-20. 